\title{
Adjacency matrix formulation of energy flow in dendrimeric polymers
}

\author{
David L. Andrews and Shaopeng Li \\ Nanostructures and Photomolecular Systems, School of Chemical Sciences, University of East \\ Anglia, Norwich NR4 7TJ, U.K.
}

\begin{abstract}
Dendrimers are synthetic, highly branched polymers with an unusually high density of chromophores. As a result of their extremely high absorption cross-sections for visible light, they represent some of the most promising new materials for energy harvesting. Although the signature of the bonding structure in dendrimers is an essentially fractal geometry, the three-dimensional molecular folding of most higher generation materials results in a chromophore layout that is more obviously akin to concentric spherical shells. The number of chromophores in each shell is a simple function of the distance from the central core. The energy of throughput optical radiation, on capture by any of the chromophores, passes by a multi-step but highly efficient process to the photoactive core. Modeling this crucial migration process presents a number of challenges. It is far from a simple diffusive random walk; each step is subject to an intricate interplay of geometric and spectroscopic features. In this report, the first results of a new approach to the theory is described, developed and adapted from an adjacency matrix formulation. It is shown how this method offers not only kinetic information but also insights into the typical number of steps and the patterns of internal energy flow.
\end{abstract}

Keywords: Dendrimers, energy harvesting, solar energy, nanoarrays, modeling

\section{INTRODUCTION}

Light-harvesting dendrimers - repeatedly branched polymers supporting a multitude of chromophores - are currently a subject of keen scientific interest, and much effort is being expended on their design, synthesis, and characterization. ${ }^{1-11}$ Often built around a fluorescent core, successive generations of branches generally have a common structural motif, giving a fractal quality to the polymer. Through internal energy transfer mechanisms that are determined by a complex interplay of structural and spectroscopic features, ${ }^{12-14}$ many such materials can capture and harness the energy of solar radiation with high efficiency, emulating principles that operate in the molecular apparatus of photosynthetic biology. The study of dendrimers has also led to a variety of other applications, e.g. dendrimer-based molecular sensors and nonlinear optical materials. ${ }^{15-18}$

To elicit details of the principles and mechanisms that govern the flow of excitation energy between chromophores, following the capture of optical energy in a dendrimeric system, the methods of quantum electrodynamics have been successfully applied in a number of recent studies, addressing both the optically linear and nonlinear regimes. ${ }^{19,20}$ The main focus of these studies has been the fundamental nature of excitation transfer between one chromophore and another, and identification of the constraints imposed by molecular symmetry and energetics. Not surprisingly there is considerable complexity when such methods are applied to the multi-step transfer routes in dendrimers, and when account is also taken of the host of alternative pathways available, the ensuing results can be very hard to interpret physically.

Bentz and $\mathrm{Kozak}^{7}$ have recently investigated the flow of energy within dendrimeric materials, modeling a probabilistic transfer of excitation from donors to the core as random and biased walks along the branches. All the branches have been treated as isolated with respect to excitation transfer. While this method allows new insights into several aspects of the process, and it begins to make a connection with experimental measurements - see for example references ${ }^{21,22}$, it cannot take into account some features that will become physically very significant as the system grows generation by generation. It might be acceptable to model energy flow assuming branches in isolation for up to three generations, but as the generations increase the polymer acquires an increasingly three-dimensional structure ${ }^{23,24}$; as it begins to approach spherical symmetry, the interaction between different branches can no longer be ignored. 
In seeking to improve on the current modeling of energy flow, any new method should aim to ensure the delivery of answers to key questions such as: what is the mean number of steps taken by the excitation, en route from the site of its initial capture to the core where the associated energy can be utilized? What does the distribution of such path lengths look like? How significant is the 'spectroscopic gradient' - a subtle difference in excited state energies - between successively visited chromophores? Also, does energy hopping within any of the chromophore shells play a significant role? To address these questions, and to provide a theory that is robust enough for significant further development, we here present a new method based on a simple statistical model, informed by the appropriate chemical principles, in which key variables can be values directly relevant to specific dendrimer systems.

\section{METHOD AND RESULT}

\section{One Generation Case}

The simplest type of dendrimer (Fig.1) is a first-generation polymer with just one set of donors, all directly connected to the core. We designate the multiplicity of first-generation donors graph theoretically in terms of a vertex degree $\rho$. For example in Fig. 1(a), $\rho=3$ indicates that there are three branches connected with the core. Here we assume that all the donors in that generation are the same kind of chromophore.<smiles>[10PH][20PH]</smiles>

(a)<smiles>[2H][V]([2H])([2H])[20PH3-]</smiles>

(b)<smiles></smiles>

(c)

Figure 1: First-generation dendrimers; $\rho(a)=3, \rho(b)=4, \rho(c)=5$.

The three-branched dendrimer of Fig. 1(a) is the prototypical representative of a major class of dendrimers, such as 1,3,5-phenylene-based materials. ${ }^{25}$ For any such system a simple energy transfer adjacency matrix can be set up;

$$
\begin{aligned}
& \begin{array}{llll}
D_{1} & D_{2} & D_{3} & A
\end{array} \\
& \begin{array}{l}
D_{1} \\
D_{2}
\end{array}\left(\begin{array}{llll}
r & f & f & a \\
f & r & f & a
\end{array}\right) \text {, } \\
& \begin{array}{l|llll}
D_{3} & f & f & r & a
\end{array} \\
& \text { A }\left(\begin{array}{llll}
0 & 0 & 0 & m
\end{array}\right)
\end{aligned}
$$

where $r$ and $m$ designate energy storage in the donor and acceptor core respectively, $f$ signifies energy transfer forward or backward between the donors, and $a$ means the core obtains the energy from one of the donors. In this matrix, each complete row represents a probability that one particular chromophore can do something to other chromophores; for example, the first row signifies that the donor $D_{1}$ retains the energy in itself, or transfers its energy to other donors, or to the core. Thus for each of the three donor rows, we have the relation $r+2 f+a=1$, expressing energy conservation. In the bottom row, we allow $m$ to differ from unity in order to reflect the losses that begin to occur following excitation of the core. Similarly, each column represents the actions of the donors and the acceptor on a single chromophore, e.g. the first column shows that the donors and acceptor can transfer energy back to $D_{1}$. Here we assume that energy transfer from the core back to any of the donors is negligible on account of the spectroscopic gradient (Fig. 2). ${ }^{26,27}$ Allowing for the possibility of energy transfer between donors in the same generation is an important feature of our model. Although 


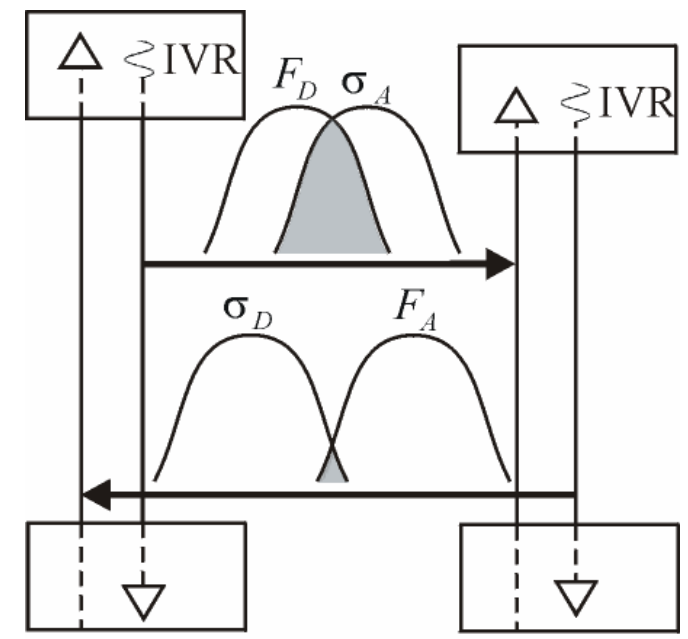

$\mathrm{D}$

A

Figure 2: Directing influence of a spectroscopic gradient on resonance energy transfer from D to A. Dotted lines indicate the range of vibrational energy levels that give rise to the emission and absorption profiles. The strong overlap of the donor emission and acceptor absorption spectra (shaded area in the upper graph) indicates that energy transfer is favorable. After the transfer, internal vibrational relaxation (IVR) in the acceptor shifts its emission spectrum to a slightly lower frequency than its absorption counterpart. As the donor absorption profile has a slightly higher frequency than its emission spectrum (again due to IVR), back-transfer from A to D is inhibited by the much smaller overlap of their respective emission and absorption profiles (lower graph). The acceptor is extremely unlikely to transfer energy back to the donor, due to the reduced spectral overlap

the different donors are not chemically bonded, they can undergo Förster energy transfer between themselves even if the associated rate is small. Such effects will generally grow in significance with the multiplicity $\rho$; using the same method, we can readily obtain the matrices for molecules with four, five, or more branches.

To interrogate the flow of energy following initial excitation, we consider repeated operations of the matrix (1) on a an initial state vector signifying that one of the donors has been excited:

$$
\left(\begin{array}{llll}
1 & 0 & 0 & 0
\end{array}\right) \text {. }
$$

Again taking in Fig. 1(a) as an example, operating with matrix (1) on the row matrix (2) generates the result;

$$
\left(\begin{array}{llll}
r & f & f & a
\end{array}\right) \text {. }
$$

The last element in this row matrix shows the proportion of the excitation transferred to the core after one iteration, which we can denote as;

$$
P_{1}=a
$$

Similarly, we can also implement a second iteration, a third, and in general the $n^{\text {th }}$ by using the method above. For the three-branched dendrimer the proportion of the excitation that transfers in the $n^{\text {th }}$ step, is thus:

$$
P_{n}=a \sum_{i=0}^{n-1}(r+2 f)^{n-1-i} m^{i} .
$$

Thus we secure the following result for the proportion of excitation that has transferred to the core, after the $n^{\text {th }}$ step;

$$
Q_{n}=a \sum_{n=1}^{\infty} \sum_{i=0}^{n-1}(r+2 f)^{n-1-i} m^{i}
$$


The above result is in fact generally applicable to first generation dendrimers of threefold or higher symmetry. To illustrate its application it is expedient to simplify the case, assuming $r+2 f=m$ (this condition is physically reasonable, although here we use it purely for convenience). Eqn (5) now takes its simplest form;

$$
P_{n}=a \times n \times m^{n-1} .
$$

We illustrate the behavior in the figures below, taking $m=0.9$. Fig. 3 shows the proportion of excitation as a function of the iteration count; from the graph we observe that the energy transfer becomes most effective at around $n=9,10$. The full development of energy transfer is exhibited in Fig. 4, based on:

$$
Q_{n}=\sum_{n=1}^{\infty} a \times n \times m^{n-1} .
$$

From the latter graph we observe that after about 65 to 70 steps the energy transfer essentially reaches completion, and the system becomes energetically stable.

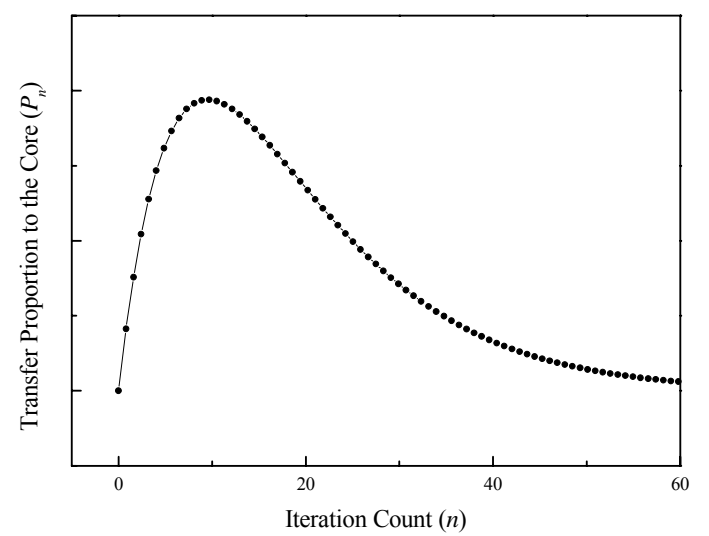

Figure 3: Transfer proportion plotted against iteration count (one generation case)

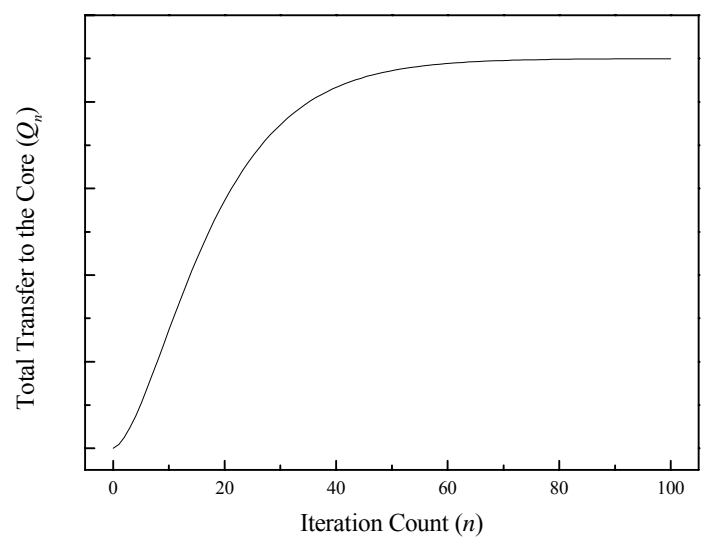

Figure 4: Total transfer plotted against the iteration count (one-generation case). 


\section{Two-Generation Case}

We now develop theory for two-generation dendrimers, taking molecule (a) of Fig. 1 as an example. Every donor of the first generation extends two new branches forming the second generation (Fig.5). The chromophores of the new generation could be chemically identical to those in the first generation, but we shall allow them to be different for the sake of generality. Using a method similar to the case described above, it is apparent that the transfer process must now be described by a $10 \times 10$ transfer matrix, equation (9).

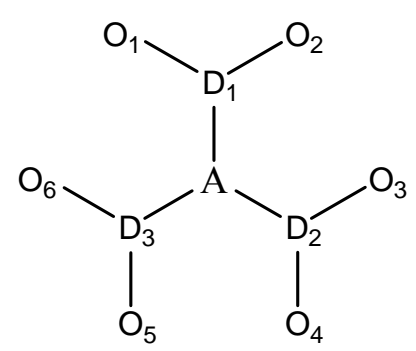

Figure 5: Second generation $\rho=3$ dendrimer.

\begin{tabular}{|c|c|c|c|c|c|c|c|c|c|c|}
\hline & $O_{1}$ & $\mathrm{O}_{2}$ & $\mathrm{O}_{3}$ & $\mathrm{O}_{4}$ & $O_{5}$ & $\mathrm{O}_{6}$ & $D_{1}$ & $D_{2}$ & $D_{3}$ & $A$ \\
\hline$O_{1}$ & $r$ & $f$ & 0 & 0 & 0 & 0 & $f^{\prime}$ & 0 & 0 & 0 \\
\hline $\mathrm{O}_{2}$ & $f$ & $r$ & 0 & 0 & 0 & 0 & $f^{\prime}$ & 0 & 0 & 0 \\
\hline $\mathrm{O}_{3}$ & 0 & 0 & $r$ & $f$ & 0 & 0 & 0 & $f^{\prime}$ & 0 & 0 \\
\hline$O_{4}$ & 0 & 0 & $f$ & $r$ & 0 & 0 & 0 & $f^{\prime}$ & 0 & 0 \\
\hline$O_{5}$ & 0 & 0 & 0 & 0 & $r$ & $f$ & 0 & 0 & $f^{\prime}$ & 0 \\
\hline$O_{6}$ & 0 & 0 & 0 & 0 & $f$ & $r$ & 0 & 0 & $f^{\prime}$ & 0 \\
\hline$D_{1}$ & $f^{\prime \prime}$ & $f^{\prime \prime}$ & 0 & 0 & 0 & 0 & $r^{\prime}$ & $d$ & $d$ & $a$ \\
\hline$D_{2}$ & 0 & 0 & $f^{\prime \prime}$ & $f^{\prime \prime}$ & 0 & 0 & $d$ & $r^{\prime}$ & $d$ & $a$ \\
\hline$D_{3}$ & 0 & 0 & 0 & 0 & $f^{\prime \prime}$ & $f^{\prime \prime}$ & $d$ & $d$ & $r^{\prime}$ & $a$ \\
\hline$A$ & 0 & 0 & 0 & 0 & 0 & 0 & 0 & 0 & 0 & $m)$ \\
\hline
\end{tabular}

where $f^{\prime}$ signifies excitation transfer from the peripheral generation to the inner generation, while $f^{\prime \prime}$ denotes transfer back to the outer generation from the inner; $r^{\prime}$ represents excitation retained by the inner generation and $d$ the case of excitation transfer between first generation chromophores; all other terms are as defined previously. In the above matrix, it is only possible for transfer to occur between peripheral donors when they are nearest neighbors $\left(O_{1}\right.$ with $O_{2}$, $\mathrm{O}_{3}$ with $\mathrm{O}_{4}, \mathrm{O}_{5}$ with $\mathrm{O}_{6}$ ); other inter-donor transfers are negligible because of the much larger associated distances. ${ }^{28,29}$ Continuing, the row matrix for the initial excitation is:

$$
\left(\begin{array}{llllllllll}
1 & 0 & 0 & 0 & 0 & 0 & 0 & 0 & 0 & 0
\end{array}\right) \text {. }
$$

For simplification, we now assume that because of the orientation of the chromophores, only the transfer of excitation between adjacent layers is allowed. Physically, one can argue that transfer from inside layer to outside layer is forbidden because of the spectral overlap; also we ignore transfer between chromophores of the same generation, that is, all the $f, f^{\prime \prime}$, and $d$ elements in matrix (9) are equated to zero; $f^{\prime}$ elements are then relabeled as $f$. Since the calculations of the above matrices are quite complicated, we now utilize a block matrix method here to make it easier. The simplified matrix then becomes;

$$
\left(\begin{array}{ccc}
R & F & 0 \\
0 & R^{\prime} & A \\
0 & 0 & m
\end{array}\right)
$$


where the individual block matrices in Eqn (11) are defined as follows;

$$
R=\left(\begin{array}{llllll}
r & 0 & 0 & 0 & 0 & 0 \\
0 & r & 0 & 0 & 0 & 0 \\
0 & 0 & r & 0 & 0 & 0 \\
0 & 0 & 0 & r & 0 & 0 \\
0 & 0 & 0 & 0 & r & 0 \\
0 & 0 & 0 & 0 & 0 & r
\end{array}\right), \quad F=\left(\begin{array}{ccc}
f & 0 & 0 \\
f & 0 & 0 \\
0 & f & 0 \\
0 & f & 0 \\
0 & 0 & f \\
0 & 0 & f
\end{array}\right), \quad R^{\prime}=\left(\begin{array}{ccc}
r^{\prime} & 0 & 0 \\
0 & r^{\prime} & 0 \\
0 & 0 & r^{\prime}
\end{array}\right), \quad A=\left(\begin{array}{l}
a \\
a \\
a
\end{array}\right) .
$$

Then the initial matrix also changes to;

$$
\left(\begin{array}{lll}
1 & 0 & 0
\end{array}\right) \text {. }
$$

Doing the calculation, we know that for the first iteration of the step transfer, there is no excitation flow into the core. The excitation begins to flow into the core from the second step. We therefore denote the second iteration as the first step in our calculation. Using the same method with which we obtained the general form for the one-generation case, we can obtain the following general form for the two-generation case;

$$
P_{n}=F A \sum_{i=0}^{n-1} \sum_{j=0}^{n-i-1} m^{i} R^{n-i-j-1} R^{\prime j} .
$$

Similarly, the total excitation transferred into the core we obtain;

$$
Q_{n}=F A \sum_{n=1}^{\infty} \sum_{i=0}^{n-1} \sum_{j=0}^{n-i-1} m^{i} R^{n-i-j-1} R^{\prime j}
$$

Finally, we note that by setting $r=r^{\prime}=m$, calculation delivers the following result;

$$
P_{n}=F A \times \frac{n(n+1)}{2} \times m^{n-1} .
$$

The resulting transfer graphs are shown in Figs 6 and 7. From Fig. 6, we observe that the highest probability of transfer happens at the $18^{\text {th }}$ or the $19^{\text {th }}$ step (which, considering the first transfer to the core is zero, means that the final result should represent the $19^{\text {th }}$ or the $20^{\text {th }}$ step). Similarly, the total excitation transferred in two-generation case, illustrated in Fig. 7, shows that we can expect to find that the transfer approaches completion after about 106 steps.

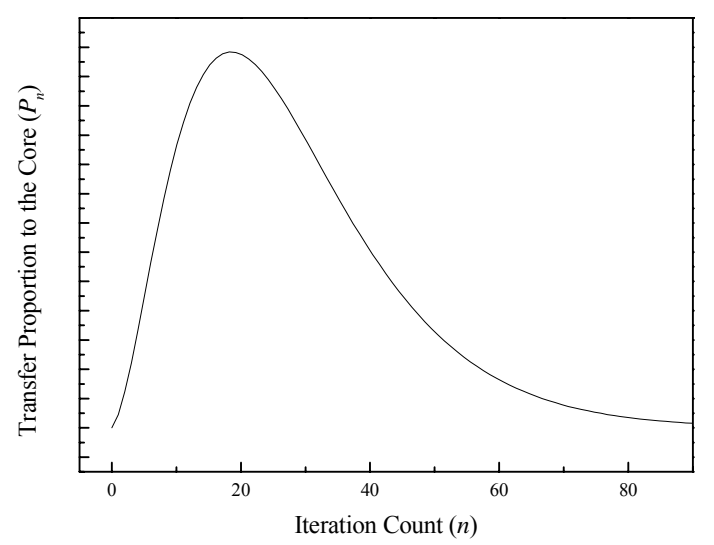

Figure 6: Transfer proportion plotted against transfer count (two-generation case). 


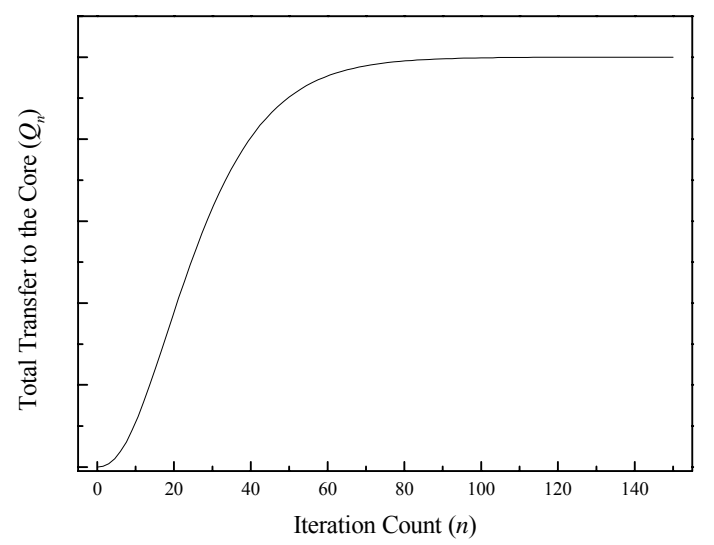

Figure 7: Total excitation transfer plotted against transfer count (two-generation case).

\section{DISCUSSION AND CONCLUSION}

In this paper we have introduced a new theoretical model for the study of excitation energy flow within dendrimeric molecules, following photoexcitation. Compared to existing theories our model strikes out in a new direction, casting the flow of excitation using a simple statistical model. Here exemplified by simple low-generation molecules, the model is robust, and it is one that is applicable to systems of arbitrarily high symmetry and generation number. It is a method that can be developed with direct reference to significant chemical and physical features, such as bonding, spectral overlap, transfer distance etc; it can also deliver quantitative results based on the input of spectroscopic and structural data. At a later stage in the analytical development our quantum electrodynamical calculations will supply such details in generic form.

Before concluding it is reasonable to enquire whether any information is lost by dealing, as we do, with populations rather than quantum amplitudes. The proximity matrix approach is certainly amenable to a formulation in terms of the latter, at the price of considerably greater complexity. A related question is whether, given systems with a multiplicity of chemically equivalent chromophores, excitonic delocalization can be expected to lead to significant departures from the behavior predicted on the basis of localized channels for excitation migration. Similar questions have been rife in past debates over the precise mechanisms of energy transfer in photosynthetic systems. Here we note that even if donor lifetimes allow, the formation of symmetry-adapted exciton states will not at all influence the form of results unless there is sufficient splitting of degeneracy for individual exciton components to be separately and identifiably populated by the initial excitation - then, of course, a quantum amplitude approach ought to be employed to properly model the ensuing kinetics. However, since that experimental situation is exceptional, our method is generally valid.

We also note that symmetry-based equivalences between chromophores, a basic requirement for exciton formation, are less and less common as the dendrimer generations increase and three-dimensional folding commences. Moreover, as the number of generations grows for example to five generations, then with the onset of folding into almost spherical structures, it becomes necessary to allow for excitation transfer between the numerous peripheral donors in different branches. Again, attention should be given to a representation of fluorescence losses from the system, since these may be expected to become significant when a large number of transfer steps takes place. All such effects are readily accommodated in our own model, and they are features that we plan to explore in future work. Although the present report addresses only one- and two-generation models, we are working towards higher generation cases. Meanwhile, we are also putting quantum electrodynamical and spectral overlap factors into our transfer matrices to do some more detailed modeling.

To conclude: in this preliminary study we have shown for both one- and two-generation dendrimers that the key kinetic features of donor decay and acceptor excitation are faithfully represented by graphical output from our 
model calculations based on transfer matrices. The method generates results that are directly applicable to various types of artificial photosystem; they are also indicative of features that can be anticipated in more highly symmetric bacterial photosynthetic arrays. The presented results are mimetic of the overall kinetics of excitation transfer from the periphery to the core; in this sense each iteration represents a small and finite but abstract element of time. Clearly a registration with future experimental measurements will give a more clearly defined meaning to the elementary time interval.

\section{ACKNOWLEDGEMENT}

We are pleased to acknowledge useful discussions and contributions from Jo Slota, working in the UEA Quantum Electrodynamics Group thanks to a Nuffield Foundation Research Bursary. We also thank Richard Crisp for producing Figure 2 .

\section{REFERENCES}

1. Bar-Haim A, Klafter J, Kopelman R, "Dendrimers as Controlled Artificial Energy Antennae,” J. Am. Chem. Soc. 119 (1997), 6197

2. Bar-Haim A, Klafter J, "Geometric versus Energetic Competition in Light Harvesting by Dendrimers," J. Phys. Chem. B 102 (1998), 1662

3. Bar-Haim A, Klafter J, "Dendrimers as light harvesting antennae," J. Lumin. 76, 77 (1998), 197

4. Poliakov EY, Chemyak V, Tretiak S, Mukamel S, "Exciton-scaling and optical excitations of self-similar phenylacetylene dendrimers," J. Chem. Phys. 110 (1999), 8161

5. Minami T, Tretiak S, Chernyak V, Mukamel S, "Frenkel-exciton Hamiltonian for dendrimeric nanostar," J. Lumin. 87-89 (2000), 115

6. Andrews DL, Bradshaw DS, “Optically nonlinear energy transfer in light-harvesting dendrimers,” J. Chem. Phys. 121 (2004) 2445

7. Bentz JL, Kozak JJ, "Influence of geometry on light harvesting in dendrimeric systems. II. nth-nearest neighbour effects and the onset of percolation," J. Lumin. 121 (2006), 62

8. Tran ML, Zvyagin AV, Plakhotnik T, "Synthesis and spectroscopic observation of dendrimer-encapsulated gold nanoclusters," Chem. Comm. 22 (2006), 2400

9. Kinberger GA, Taulane JP, Goodman M, "The design, synthesis, and characterization of a PAMAM-based triple helical collagen mimetic dendrimer," Tetrahedron 62 (2006), 5280

10. Yamazaki N, Washio I, Shibasaki Y, Ueda M, "Facile synthesis of aryl ether dendrimer from unprotected AB(2) building blocks using thionyl chloride as an activating agent," Org. Lett. 8 (2006), 2321

11. Hoover NN, Auten BJ, Chandler BD, "Tuning supported catalyst reactivity with dendrimer-templated Pt-Cu nanoparticles," J. Phys. Chem. B 110 (2006), 8606

12. Thomas KRJ, Thompson AL, Sivakumar AV, Bardeen CJ, Thayumanavan S, "Energy and Electron Transfer in Bifunctional Non-Conjugated Dendrimers," J. Am. Chem. Soc. 127 (2005), 373

13. Supritz C, Engelmann A, Reineker P, "Energy transport in dendrimers," J. Lumin. 119-120 (2006), 337

14. Akai I, Okada A, Kanemoto K, Karasawa T, Hashimoto H, Kimura M, "Quenching of energy transfer by freezing molecular vibrations in light-harvesting small dendrimer," J. Lumin. 119 (2006) 283

15. Fréchet JMJ, "Dendrimers and other dendritic macromolecules: From building blocks to functional assemblies in nanoscience and nanotechnology," J. Polymer Sci. A: Polymer Chem. 41 (2003) 3713

16. Flomenbom O, Klafter J, Amir RJ, Shabat D, "Dendrimer-based devices: Antennae and amplifiers," pp. 245-279 in Energy Harvesting Materials, ed. Andrews DL (World Scientific, New Jersey 2005)

17. D'Abruoso GD, McGrath DV, "Energy harvesting in synthetic dendritic materials," pp. 281-334 in Energy Harvesting Materials, ed. Andrews DL (World Scientific, New Jersey 2005)

18. Peng Z, Melinger JS, Kleiman V, "Light harvesting unsymmetrical conjugated dendrimers as photosynthetic mimics," Photosynthesis Research 87 (2006), 115

19. Jenkins RD, Andrews DL, "Exciton resonance energy transfer: Effects of geometry and transition moment orientation in model photosystems," Photochem. Photobiol. Sci. 2 (2003), 130

20. Jenkins RD, Andrews DL, "Multichromophore excitons and resonance energy transfer: Molecular quantum electrodynamics," J. Chem. Phys. 118 (2003), 3470 
21. Varnavski O, Samuel IDW, Palsson LO, Beavington R, Burn PL, Goodson T, "Investigations of excitation energy transfer and intramolecular interactions in a nitrogen corded distrylbenzene dendrimer system," J. Chem. Phys. 116 (2002), 8893

22. Lahankar SA, West R, Varnavski O, Xie XB, Goodson T, Sukhomlinova L, Twieg R, "Electronic interactions in a branched chromophore investigated by nonlinear optical and time-resolved spectroscopy," J. Chem. Phys. 120 (2004), 337

23. Tomalia DA, Naylor AM, Goddard WA, "Starburst Dendrimers: Molecular-Level Control of Size, Shape, Surface Chemistry, Topology, and Flexibility from Atoms to Macroscopic Matter," Angew. Chem. Int. Ed. Eng. 29 (1990), 138

24. Thayumanavan S, Bharathi P, Sivanandan K, Vutukuri DR, "Towards dendrimers as biomimetic macromolecules," C. R. Chimie 6 (2003), 767

25. Miller TM, Neenan TX, Zayas R, Bair HE, "Synthesis and characterization of a series of monodisperse, 1,3,5phenylene-based hydrocarbon dendrimers including $\mathrm{C}_{276} \mathrm{H}_{186}$ and their fluorinated analogs," J. Am. Chem. Soc. 114 (1992), 1018

26. Shortreed MR, Swallen SF, Shi ZY, Tan WH, Xu ZF, Devadoss C, Moore JS, Kopelman R, "Directed energy transfer funnels in dendrimeric antenna supermolecules," J. Phys. Chem. B 101 (1997), 6318

27. Andrews DL and Crisp RG, "Principles of Directed Electronic Energy Transfer", Springer Series on Fluorescence, 4 (2006) in press

28. Andrews DL, Juzeliūnas G, "Intermolecular energy transfer: retardation effects," J. Chem. Phys. 96 (1992), 6606

29. Andrews DL, Allcock P, "Biomolecular photophysics," Chem. Soc. Rev. 24 (1995), 259 